\title{
GERMINAÇÃO E INCIDÊNCIA DE FUNGOS EM TESTES COM SEMENTES DE Brachiaria brizantha Stapf. ${ }^{1}$
}

\author{
D.C.F.S. DIAS \\ Eng ${ }^{a}$ Agr ${ }^{a}$, M.Sc., EMCAPA/Estą̧ão Experimental de Linhares, Caixa Postal, 62 - CEP: 29900-970-Linhares, ES. \\ F.F. DE TOLEDO \\ Prof. Titular Aposentado do Dep. de Agricultura da ESALQ/USP - Caixa Postal, 9 - CEP: 13418-900-Piracicaba,SP.
}

\begin{abstract}
RESUMO: O presente trabalho teve por objetivo estudar o efeito da escarificação com ácido sulfúrico e da aplicação de fungicidas (thiabendazol, captan, thiram e iprodione + thiram) nos resultados dos testes de germinação e no desenvolvimento de microrganismos sobre sementes de $\boldsymbol{B}$. brizantha. Além dos testes de germinaçāo, os quais foram avaliados quanto à incidência de microrganismos aos 10 e 21 dias, conduziram-se testes de sanidade, procurando-se identificar a microflora presente. A analise dos dados e interpretaçāo dos resultados permitiram as seguintes conclusões: a escarificação com o ácido não promoveu acréscimo significativo na germinação, tendo contribuído para a redução do nível de incidência de fungos ao se comparar com a testemunha. Os fungicidas aplicados sobre as sementes escarificadas contribuiram para uma melhor germinação e para a redução na ocorrência de microrganismos, destacando-se a mistura iprodione + thiram. $O$ uso dos fungicidas também facilitou a interpretação dos testes de germinação. Foram encontrados os seguintes fungos: Alternaria tenuis, Aspergillus spp., Cladosporium sp., Curvularia sp., Drechslera sp.p, Epicoccum sp., Fusarium sp., Neurospora monilia, Penicillium sp., Phoma sp., Rhizopus sp., Tricholhecium sp. e Trichoconiella padwikii.

Descritores: Brachiaria brizantha, sementes, germinação, escarificação, fungos, fungicidas.
\end{abstract}

\section{GERMINATION AND FUNGI INCIDENCE IN TESTS WITII Brachiaria brizantha Stapf. SEEDS}

\begin{abstract}
The present research was carried out to evaluate the effects of sulphuric acid scarification and fungicide (thiabendazol, captan, thiram and iprodione + thiram) applications on germination tests of $B$. brizantha and on mycrflora development associated with those tests. In germination tests mycoflora were identified and evaluated on the $10^{\text {th }}$ and the $21^{*}$ day; at the same time blotter tests were carried out to study the microflora. Interpretations of the results showed that sulphuric acid scarification did not increase significantly the germination but checked fungi development and showed less efficiency than fungicides. Among these the mixture of iprodione and thiram provided the best results. The following fungi were identified: Allernaria tenuis, Aspergillus spp., Cladosporium sp., Curvularia sp., Drechslera spp., Epicoccum sp., Fusarium sp., Neurospora monilia, Penicillium sp., Phoma sp., Rhizopus sp., Trichothecium sp. e Trichoconiella padwikii.
\end{abstract}

Key Words: Brachiaria brizantha, seeds, germination, scarification, fungi, fungicides.

\section{INTRODUÇÃO}

A Brachiaria brizantha Stapf.. é uma forrageira que tem grande importância na formação de pastagens melhoradas em diversas regiões do País. O sucesso da implantação dessas pastagens depende, em grande parte, do valor cultural das sementes utilizadas.

Dentre os fatores que contribuem para o baixo valor cultural, comumente apresentado pelas sementes desta espécie, destaca-se a presença de dormência pós-colheita. Nos testes de germinação em laboratório, vem sendo utilizado o ácido sulfúrico concentrado, associado à alternância de temperatura e de luz e à presença de solução de nitrato de potássio no substrato, para superar a dormência das sementes de várias espécies de braquiária, conforme instruções encontradas nas Regras Australianas para Análise de Sementes (AUSTRÁLIA, 1970), nas Regras Internacionais para Análise de Sementes (INTERNATIONAL SEED TESTING ASSOCIATION, 1985) e de acordo com as recomendações de ORTOLANI (1989).

1 Parte da dissertação apresentada pelo primeiro autor à Escola Superior de Agricultura "Luiz de Queiroz" da Universidade de São Paulo, para obtenção do lítulo de Mestre em Fitotecnia. 
Pesquisas realizadas por DAVIDSON (1966), GROF (1968) e GOEDERT (1985) mostraram a eficiência da aplicação desse composto químico em $B$. decumbens, ao contrário dos resultados obtidos por JARK FILHO (1976), TOSELLO \& ATALLA (1978) e WHITEMAN \& MENDRA (1982). Por sua vez, MCLEAN \& GROF (1968) e GOEDERT (1985) revelaram que a eficiência da escarificação de sementes de braquiária com ácido sulfúrico pode apresentar resultados variáveis conforme a espécie.

Embora tal tratamento venha se mostrando eficiente, tem sido relatado que os testes de germinação com sementes escarificadas com ácido sulfúrico exibem, invariavelmente, intensa ocorrência de microrganismos, tanto nas sementes como no substrato, dificultando a interpretação e avaliação das plântulas e, possivelmente, exercendo influência sobre o resultado final da análise.

A importância de patógenos associada a sementes já se acha comprovada pela pesquisa (NEERGAARD, 1977), mas são escassas as informações a respeito da qualidade sanitária das sementes de forrageiras utilizadas pelos pecuaristas. URBEN (1987), em levantamento feito em sementes de 24 gêneros de gramíneas forrageiras, constatou elevado número de espécies de microrganismos, sendo que, em Brachiaria spp., foram identificados os gêneros Fusarium, Cladosporium, Penicillium, Phyllosticta, Curvularia, Drechslera, Pyrenochaeta, Rhizopus, Epicoccum e Aspergillus. MENDES et al. (1989) observaram, em sementes de braquiária, os gêneros Drechslera, Phoma e Curvularia. Por sua vez, ANDERSEN (1955; 1957) e McGEE (1979) realizaram pesquisas com o objetivo de avaliar a influência de microrganismos sobre os resultados dos testes de germinação em laboratório.

A utilização de produtos químicos para a desinfecção e proteção das sementes tem sido uma prática bastante difundida em diversas espécies. No entanto, até o presente momento, não existe preocupação em relação ao tratamento de sementes de gramíneas forrageiras, salvo nos trabalhos de LUTRELL et al. (1955), PANDY et al. (1981), SHETTY et al. (1982), PANDY \& GUPTA (1984), KONDE et al. (1984) e TOLEDO (1977).

Pelos motivos expostos, o presente trabalho teve como objetivos estudar o efeito da escarificação química com ácido sulfúrico e da aplicação de fungicidas nos resultados de testes de germinação e identificar os microrganismos associados às sementes de $B$. brizantha.

\section{MATERIAL E MÉTODOS}

Sementes de B. brizantha Stapf. cultivar Marandu, colhidas em julho de 1987 (lote 1) e julho de 88 (lote 2), pelo método de varredura, foram utilizadas para a realização da parte experimental, em quatro épocas (lote 1) e três épocas (lote 2), a saber: fevereiro, maio, agosto e novembro de 1989.

Após a recepção, procedeu-se à homogeneização e divisão dos lotes em divisor de solos, obtendo-se, assim, uma amostra de, aproximadamente, 500 gramas de cada lote. Cada uma delas foi ventilada em soprador "South Dakota", utilizando-se abertura 26, para eliminação de espiguetas vazias e impurezas leves. Em seguida, por separação visual, removeram-se todas as impurezas restantes para se obter apenas sementes puras.

A amostra resultante, de cerca de 300 gramas de sementes puras, foi novamente homogeneizada e dividida, obtendo-se seis subamostras de cerca de 50 gramas cada. Cinco destas foram submetidas à escarificação com ácido sulfúrico e a remanescente foi reservada, constituindo-se a testemunha sem escarificação.

Para a realização da escarificação, as sementes foram imersas em ácido sulfúrico concentrado comercial por 13 minutos, lavadas em água corrente para eliminar todo o ácido $\mathrm{e}, \mathrm{em}$ seguida, mantidas por 60 minutos em recipientes contendo $200 \mathrm{ml}$ de água; finalmente, foram colocadas para secar à sombra. Depois de secas, quatro das subamostras escarificadas foram destinadas à aplicação dos fungicidas thiabendazol, captan, thiram e iprodione + thiram, na dose de $200 \mathrm{~g}$ de produto comercial por $100 \mathrm{Kg}$ de sementes. Obtiveram-se, assim, os tratamentos que se encontram expostos na Figura 1.

Todas as subamostras que formaram os seis tratamentos foram submetidas ao teste de germinação, em 4 repetições de 50 sementes, realizado em gerbox contendo duas folhas de papel germibox, previamente esterilizadas em estufa a $105^{\circ} \mathrm{C} / 30$ minutos, umedecidas com $13 \mathrm{ml}$ de solução de $\mathrm{KNO}_{3}$ a $0,2 \%$ e colocadas para germinar em aparelho STULTS, utilizando-se temperatura alternada de $20-35^{\circ} \mathrm{C}$, proporcionando iluminação durante o período de 8 horas, associada à temperatura mais alta, conforme instruções das RIAS (INTERNATIONAL SEED TESTING ASSOCIATION, 1985). A duração do teste foi de 21 dias, efetuando-se contagens a cada sete dias. 
Os testes de germinação foram avaliados quanto às suas condições sanitárias no $10^{\circ}$ e $21^{\circ}$ dia após a instalação. Além disso, a avaliação sanitária dos seis tratamentos de cada lote foi realizada através do método do papel de filtro, utilizando-se 8 repetições de 25 sementes por tratamento, conforme metodologia descrita por NEERGAARD (1977). A identificação das estruturas reprodutivas dos microrganismos foi feita de acordo com BARNETT \& HUNTER (1972).

Os dados obtidos dos testes de germinação e de sanidade, nas diferentes épocas, foram submetidos à análise de variância, segundo delineamento em blocos casualizados. A comparação entre as médias foi efetuada através do teste de Tukey, ao nível de $5 \%$ de probabilidade. Foi conduzida, ainda, a análise de correlação simples entre os resultados dos referidos testes.

\section{RESULTADOS E DISCUSSÃO}

Observando-se as TABELAS 1 e 2 verifica-se que o tratamento $5\left(\mathrm{H}_{2} \mathrm{SO}_{4}\right)$ demonstrou um pequeno efeito benéfico, não significativo, sobre a porcentagem de germinação em relação à testemunha, para o lote 1 , diferença esta que, em análise de rotina, pode ser considerada expressiva. No lote 2, a aplicação de ácido sulfúrico (tratamento 5) não promoveu acréscimo na germinação. Tambćm GROF (1968) não encontrou resultados significativos para a aplicação deste ácido em sementes de $B$. decumbens, armazenadas por 10 meses.

Por sua vez, os fungicidas utilizados não mostraram efeito significativamente diferente entre si, ou seja, foram semelhantemente eficientes para proteger as sementes, nos dois lotes estudados. LUTTRELL et al. (1955) e PANDY et al. (1981) também verificaram que a aplicação de fungicidas em sementes de milheto e de Setaria ilalica, respectivamente, contribuiu para aumentar a germinação. Por outro lado, TOLEDO (1977) verificou que o uso de fungicidas facilitou a interpretação dos testes de germinação de $P$. maximum, porém, sem causar efeito sobre a capacidade germinativa.

A análise feita para se confrontar as diferentes épocas (TABELAS 1 e 2) não mostrou diferenças significativas para o lote 1 , enquanto que o lote 2 exibiu, ao longo do período experimental, um aumento significativo na germinação das sementes, o que pode ser atribuído à redução na dormência das mesmas.

Com relação à avaliação sanitária aos 10 e 21 dias do teste de germinação (Quadros 2 e 3), pode-se verificar que os tratamentos envolvendo a aplicação de fungicidas não diferiram significativamente entre si, com certo destaque para o iprodione + thiram. Observou-se, ainda, na avaliação feita aos 10 dias, que, já nesta ocasião, a presença de microrganismos era evidente, principalmente nos tratamentos 5 e 6 . Este último, apresentou maior incidência de fungos quando comparado aos demais, não diferindo estatisticamente do tratamento 5. No entanto, os dados sugerem que as sementes escarificadas com ácido e sem fungicida (tratamento 5) exibiram um bom desempenho em relação à testemunha, ou seja, o ácido promoveu uma assepsia superficial das sementes. TANAKA \& PAOLINELLI (1984), trabalhando com sementes de algodão, constataram que o deslintamento com ácido sulfúrico facilitou o exame e a deteç̧ão de patógenos, reduzindo o inóculo dos contaminantes externos presentes superficialmente no línter. Assim, provavelmente, o referido ácido atuou no sentido de reduzir o potencial de inóculo presente externamente nas espiguetas.

A utilização de fungicidas contribuiu para a redução da ocorrência de microrganismos, facilitando a interpretação do teste e as contagens das plântulas. Resultados semelhantes foram obtidos por TOLEDO (1977) em P. maximum.

Ainda nos Quadros 2 e 3, o exame dos dados referentes ao teste de sanidade permite destacar a elevada incidência de fungos na testemunha, confirmando as avaliações sanitárias dos testes de germinação. Em ambos os lotes foi possivel observar a superioridade dos tratamentos $2\left(\mathrm{H}_{2} \mathrm{SO}_{4}\right.$ captan $), 3\left(\mathrm{H}_{2} \mathrm{SO}_{4}\right.$ thiram $)$ e $4\left(\mathrm{H}_{2} \mathrm{SO}_{4}\right.$ iprodione + thiram) em relação ao tratamento 1 $\left(\mathrm{H}_{2} \mathrm{SO}_{4}\right.$ thiabendazol). Este revelou ação moderada, semelhante ao ácido sulfúrico isolado (tratamento 5), que, por sua vez, não diferiu da testemunha, no lote 1. No lote 2, os tratamentos 1,5 e 6 mostraram-se estatisticamente iguais.

Os dados revelam, ainda, que os tratamentos fungicidas contribuíram para a redução na incidência de fungos, principalmente em relação à testcmunha, destacando-se o iprodione + thiram, que possibilitou a erradicação de Drechslera spp.. no lote 1 .

Com rclação às épocas de avaliação (TABELAS 1 e 2), pode-se observar que não houve diferenças significativas entre as mesmas, 


\begin{tabular}{||c||}
\hline \multicolumn{1}{|c||}{ Tratamentos } \\
\hline 1 - Sementes escarificadas com $\mathrm{H}_{2} \mathrm{SO}_{4}$ e tratadas com thiabendazol. \\
2 - Sementes escarificadas com $\mathrm{H}_{2} \mathrm{SO}_{4}$ e tratadas com captan. \\
3 - Sementes escarificadas com $\mathrm{H}_{2} \mathrm{SO}_{4}$ e tratadas com thiram. \\
4 - Sementes escarificadas com $\mathrm{H}_{2} \mathrm{SO}_{4}$ e tratadas com iprodione + thiram. \\
5 - Sementes escarificadas com $\mathrm{H}_{2} \mathrm{SO}_{4}$. \\
6 - Sementes não escarificadas (testemunha).
\end{tabular}

Figura 1 - Quadro demonstrativo dos tratamentos aplicados às subamostras de sementes de B.brizantha. Piracicaba, 1989.

TABELA 1 - Resultados de germinação e incidência de fungos aos 10 e 21 dias do teste de germinação e no teste de sanidade de sementes de B.brizantha, lote 1: médias (\%) de 4 épocas e coeficientes de variação. Piracicaba, 1989.

\begin{tabular}{|c|c|c|c|c|}
\hline \multirow{2}{*}{ Tratamentos } & \multicolumn{4}{|c|}{ Médias } \\
\hline & Germinação & $\begin{array}{l}\text { Incidência } \\
10 \text { dias }\end{array}$ & $\begin{array}{l}\text { Incidência } \\
21 \text { dias }\end{array}$ & Sanidade \\
\hline 1. $\mathrm{H}_{2} \mathrm{SO}_{4}+$ thiabendazol & $74 \mathrm{a}$ & $3,0 \mathrm{bc}$ & $4,6 \mathrm{bc}$ & $10,3 \mathrm{bc}$ \\
\hline 2. $\mathrm{H}_{2} \mathrm{SO}_{4}+$ captan & $73 \mathrm{a}$ & $1,4 \mathrm{bc}$ & $4,6 \mathrm{bc}$ & $2,8 \mathrm{c}$ \\
\hline 3. $\mathrm{H}_{2} \mathrm{SO}_{4}+$ thiram & $77 \mathrm{a}$ & $1,1 \mathrm{bc}$ & $2,3 \mathrm{c}$ & $1,3 \mathrm{c}$ \\
\hline 4. $\mathrm{H}_{2} \mathrm{SO}_{4}+$ iprodione + thiram & $77 \mathrm{a}$ & $0,5 \mathrm{c}$ & $0.8 \mathrm{c}$ & $0,4 c$ \\
\hline 5. $\mathrm{H}_{2} \mathrm{SO}_{4}$ & $73 \mathrm{a}$ & $11,5 \mathrm{ab}$ & $17,8 \mathrm{ab}$ & $21,1 \mathrm{ab}$ \\
\hline 6. Testemunha & $51 \mathrm{a}$ & $31,0 \mathrm{a}$ & $32,4 \mathrm{a}$ & $42,4 \mathrm{a}$ \\
\hline \multicolumn{5}{|l|}{ Épocas } \\
\hline $1^{\mathrm{a}}$ & 72 a & $15,0 \mathrm{a}$ & $12,3 a$ & $16,3 \mathrm{a}$ \\
\hline $2^{\mathbf{a}}$ & $71 \mathrm{a}$ & $4,6 \mathrm{a}$ & $7,7 \mathrm{a}$ & $11,7 \mathrm{a}$ \\
\hline $3^{\mathbf{a}}$ & $74 \mathrm{a}$ & $6,4 \mathrm{a}$ & $8,1 \mathrm{a}$ & $15,3 \mathrm{a}$ \\
\hline $4^{\mathrm{a}}$ & $72 \mathrm{a}$ & $6,3 \mathrm{a}$ & $13,7 \mathrm{a}$ & $8,8 \mathrm{a}$ \\
\hline C.V. (\%) & 8,1 & 62,8 & 49,5 & 52,4 \\
\hline
\end{tabular}

As médias seguidas pela mesma letra não diferiram entre si, pelo teste de Tukey, ao nível de $5 \%$ de probabilidade. 
TABELA 2 - Resultados de germinação e incidência de fungos aos 10 e 21 dias do teste de germinação e no teste de sanidade de sementes de B.brizantha, lote 2: médias (\%) de 3 épocas e coeficientes de variação. Piracicaba, 1989.

\begin{tabular}{lcccc}
\hline \hline & \multicolumn{4}{c}{ Médias } \\
\cline { 2 - 5 } Tratamentos & Germinação & $\begin{array}{c}\text { Incidência } \\
10 \text { dias }\end{array}$ & $\begin{array}{c}\text { Incidência } \\
21 \text { dias }\end{array}$ & Sanidade \\
\hline 1. $\mathrm{H}_{2} \mathrm{SO}_{4}+$ thiabendazol & $64 \mathrm{a}$ & $6,0 \mathrm{abc}$ & $12,5 \mathrm{abc}$ & $23,0 \mathrm{a}$ \\
2. $\mathrm{H}_{2} \mathrm{SO}_{4}+$ captan & $67 \mathrm{a}$ & $2,0 \mathrm{bc}$ & $5,2 \mathrm{bcd}$ & $1,7 \mathrm{~b}$ \\
3. $\mathrm{H}_{2} \mathrm{SO}_{4}+$ thiram & $58 \mathrm{a}$ & $1,2 \mathrm{bc}$ & $5,2 \mathrm{bcd}$ & $0,0 \mathrm{~b}$ \\
4. $\mathrm{H}_{2} \mathrm{SO}_{4}+$ iprodione + thiram & $73 \mathrm{a}$ & $0,2 \mathrm{c}$ & $0.7 \mathrm{~cd}$ & $0,0 \mathrm{~b}$ \\
$5 . \mathrm{H}_{2} \mathrm{SO}_{4}$ & $55 \mathrm{a}$ & $10,8 \mathrm{ab}$ & $17,8 \mathrm{ab}$ & $29,3 \mathrm{a}$ \\
6. Testemunha & $55 \mathrm{a}$ & $15,8 \mathrm{a}$ & $24,5 \mathrm{a}$ & $53,8 \mathrm{a}$ \\
\hline$\dot{E}_{\text {pocas }}$ & & & & $17,0 \mathrm{a}$ \\
\hline $1^{\mathrm{a}}$ & $53 \mathrm{~b}$ & $5,4 \mathrm{a}$ & $12,7 \mathrm{a}$ & $26,8 \mathrm{a}$ \\
$2^{\mathrm{a}}$ & $64 \mathrm{ab}$ & $7,1 \mathrm{a}$ & $8,1 \mathrm{a}$ & $10,2 \mathrm{a}$ \\
\hline $3^{\mathrm{a}}$ & $69 \mathrm{a}$ & $5,4 \mathrm{a}$ & $12,2 \mathrm{a}$ & 32,6 \\
\hline C.V. $(\%)$ & 9,7 & 46,6 & 32,1 & \\
\hline \hline
\end{tabular}

As médias seguidas pela mesma letra não diferiram entre si, pelo teste de Tukey, ao nível de $5 \%$ de probabiiidade.

verificando-se comportamento semelhante para os dois lotes.

Os microrganismos identificados nas avaliaçōes sanitárias dos testes de germinação para os lotes 1 e 2 encontram-se, respectivamente, nas TABELAS 3 e 4 . Além de fungos patogênicos como Drechslera spp., Fusarium sp., Phoma sp. e Curvularia sp., observou-se a ocorrência cm nívcis pouco expressivos, de fungos secundários, considerados saprófitas, tais como Epicoccum sp., Rhizopus sp. e Neurospora monilia.

Vale ressaltar que a aplicação de ácido sulfúrico nessas sementes promoveu, de modo gcral, certa redução na incidência de fungos $\mathrm{em}$ comparação com a testemunha, principalmente de uma das espécies não identificadas, de Phoma sp. e de Drechslera spp . Já os nívcis de Fusarium sp. foram praticamente mantidos no lote 1 e incrementados no lote 2, quando se utilizou tal ácido (TABELAS 3 e 4 ).
Nos testes de sanidade (TABELA 5), verificou-se a recuperação de fungos não detectados nos testes de germinação, tais como Alternaria tenuis, no lote 1 e Cladosporium sp., em ambos os lotes.

Por outro lado, Neurospora monilia e os microrganismos não identificados, presentes nos testes de germinação, não foram detectados pelo método do papel de filtro, no teste de sanidade. Isto evidencia que as condições de incubaçăo do teste $\mathrm{cm}$ questão não permitiram a recuperaçãa destes microrganismos, os quais encontraram nos testes de germinação condições propícias para a sua manifestação.

De modo geral, o teste de sanidade proporcionou maior recuperação de fungos que as avaliações sanitárias do teste de germinação, o que é perfeitamente explicável, uma vez que as condições de incubação, no caso daquele teste, são ideais ao crescimento e desenvolvimento dos fungos. 
TABELA 3 - Identificação e incidência (\%) de fungos detectados aos 10 e 21 dias, nas avaliações sanitárias dos testes de germinação de sementes de B.brizantha, lote 1: (médias das 4 épocas). Piracicaba,1989.

\begin{tabular}{|c|c|c|c|c|c|c|c|c|c|c|c|c|}
\hline \multirow{3}{*}{ FUNGOS } & \multicolumn{12}{|c|}{ INCIDÊNCIA (\%) NOS TRATAMENTOS } \\
\hline & \multicolumn{6}{|c|}{10 DIAS } & \multicolumn{6}{|c|}{21 DIAS } \\
\hline & 1 & 2 & 3 & 4 & 5 & 6 & 1 & 2 & 3 & 4 & 5 & 6 \\
\hline Aspergillus spp. & 0,3 & 0,1 & 0,0 & 0,0 & 2,6 & 0,8 & 0,4 & 0,1 & 0,0 & 0,0 & 2,8 & 0,8 \\
\hline Curvularia sp. & 0,1 & 0,0 & 0,0 & 0,0 & 0,8 & 0,1 & 0,1 & 0,0 & 0,0 & 0,0 & 0,8 & 0,1 \\
\hline Drechslera spp. & 0,9 & 0,3 & 0,5 & 0,0 & 0,9 & 5,0 & 1,5 & 0,4 & 1,4 & 0,0 & 2,4 & 6,6 \\
\hline Epicoccum sp. & 0,1 & 0,3 & 0,0 & 0,0 & 0,4 & 0,6 & 0,1 & 0,3 & 0,0 & 0,0 & 0,4 & 0,4 \\
\hline Fusarium sp. & 0,9 & 0,5 & 0,3 & 0,3 & 3,5 & 3,6 & 1,1 & 1,8 & 0,6 & 0,3 & 5,1 & 5,4 \\
\hline N. monilsa & 0.0 & 0,0 & 0,0 & 0,0 & 0,0 & 0,9 & 0,0 & 0,0 & 0,0 & 0,0 & 0,0 & 0,9 \\
\hline Penicillum sp & 0,0 & 0,1 & 0,0 & 0,0 & 0,8 & 0,5 & 0,0 & 0,1 & 0,0 & 0,0 & 0,8 & 0,5 \\
\hline Phoma sp. & 0,0 & 0,0 & 0,0 & 0,0 & 0,3 & 3,9 & 0,5 & 0,6 & 0,0 & 0,0 & 1,4 & 6,1 \\
\hline Rhizopus sp & 0,0 & 0,0 & 0,0 & 0,0 & 0,0 & 0,5 & 0,0 & 0,0 & 0,0 & 0,0 & 0,0 & 0,5 \\
\hline Nāo identif. (1) & 0.6 & 0,1 & 0,4 & 0,3 & 1,4 & 8,8 & 0,8 & 1,4 & 0,3 & 0,5 & 2,4 & 11,0 \\
\hline Não identif. (2) & 0,0 & 0.0 & 0,0 & 0,0 & 1,0 & 0,0 & 0,1 & 0,0 & 0,0 & 0,0 & 1,9 & 0,1 \\
\hline
\end{tabular}

TABELA 4 - Identificação e incidência (\%) de fungos detectados aos 10 e 21 dias, nas avaliações sanitárias dos testes de germinação de sementes de B.brizantha, lote 2: (médias das 3 épocas). Piracicaba, 1989

\begin{tabular}{|c|c|c|c|c|c|c|c|c|c|c|c|c|}
\hline \multirow{3}{*}{ FUNGOS } & \multicolumn{12}{|c|}{ INCIDÊNCIA (\%) NOS TRATAMENTOS } \\
\hline & \multicolumn{6}{|c|}{10 DIAS } & \multicolumn{6}{|c|}{21 DIAS } \\
\hline & 1 & 2 & 3 & 4 & 5 & 6 & 1 & 2 & 3 & 4 & 5 & 6 \\
\hline A. tenuss & 0,3 & 0,0 & 0,2 & 0,0 & 0,2 & 0,0 & 0,2 & 0,0 & 0,2 & 0,0 & 0,2 & 0,0 \\
\hline Aspergillus spp. & 0,2 & 0,2 & 0,0 & 0,0 & 1,0 & 0,5 & 0,5 & 0,5 & 0,0 & 0,0 & 1,2 & 1,0 \\
\hline Curvularia sp. & 0,5 & 0,0 & 0,0 & 0,0 & 0,0 & 0,2 & 0,5 & 0,0 & 0,0 & 0,0 & 0,0 & 0,2 \\
\hline Drechslera spp. & 2,7 & 0,5 & 0,2 & 0,0 & 1,8 & 7,0 & 4,7 & 2,3 & 0,5 & 0,3 & 2,3 & 9,7 \\
\hline Epicoccum sp. & 0,0 & 0,0 & 0,0 & 0,0 & 0,0 & 0,5 & 0,0 & 0,0 & 0,0 & 0,0 & 0,0 & 0,5 \\
\hline Fusarium sp. & 1,5 & 1,0 & 0,3 & 0,0 & 5,8 & 2,5 & 2,7 & 2,2 & 0,7 & 0,2 & 10,5 & 4,2 \\
\hline N. monilia & 0,0 & 0,0 & 0,0 & 0,0 & 0,0 & 1,2 & 0,2 & 0,0 & 0,0 & 0,0 & 0,0 & 3,5 \\
\hline Penicillium sp. & 0,0 & 0,0 & 0,0 & 0,0 & 0,2 & 0,0 & 0,3 & 0,0 & 0,0 & 0,0 & 0,0 & 0,0 \\
\hline Phoma sp. & 0,2 & 0,0 & 0,0 & 0,0 & 0,3 & 1,2 & 0,8 & 0,0 & 0,0 & 0,0 & 1,0 & 4,2 \\
\hline Rhizopus sp. & 0,3 & 0,0 & 0,0 & 0,0 & 0,2 & 0,7 & 1,2 & 0,0 & 0,0 & 0,0 & 0,2 & 0,7 \\
\hline Não identif. (1) & 0,3 & 0,2 & 0,2 & 0,2 & 1,3 & 3,3 & 1,7 & 0,0 & 0,7 & 0,2 & 2,2 & 4,0 \\
\hline Não identif. (2) & 0,0 & 0,2 & 0,0 & 0,0 & 0,3 & 0,0 & 0,0 & 0,2 & 0,0 & 0,0 & 0,0 & 0,0 \\
\hline
\end{tabular}

Sci. agric. Piracicaba, 50(1):68-76, fev./maio, 1993 
TABELA 5 - Identificação e incidência (\%) de fungos detectados nos tratamentos dos testes de sanidade de sementes de B.brizantha, lotes 1 e 2: (médias das épocas). Piracicaba, 1989.

\begin{tabular}{|c|c|c|c|c|c|c|c|c|c|c|c|c|}
\hline \multirow{3}{*}{ FUNGOS } & \multicolumn{12}{|c|}{ INCIDENCIA (\%) NOS TRATAMENTOS } \\
\hline & \multicolumn{6}{|c|}{ LOTE 1} & \multicolumn{6}{|c|}{ LOTE 2} \\
\hline & 1 & 2 & 3 & 4 & 5 & 6 & 1 & 2 & 3 & 4 & 5 & 6 \\
\hline A. tenuis & 0,0 & 0,0 & 0,0 & 0,0 & 0,4 & 0,1 & 0,0 & 0,0 & 0,0 & 0,0 & 0,3 & 0,0 \\
\hline Aspergillus spp. & 0,1 & 0,0 & 0,0 & 0,0 & 0,8 & 2,3 & 3,2 & 0,0 & 0,0 & 0,0 & 3,7 & 7,5 \\
\hline Cladosporium sp & 0,0 & 0,0 & 0,0 & 0,0 & 0,3 & 0,0 & 0,3 & 0,0 & 0,0 & 0,0 & 0,7 & 0,0 \\
\hline Curvularia spp. & 1,5 & 0,0 & 0,0 & 0,0 & 1,9 & 4,1 & 0,3 & 0,0 & 0,0 & 0,0 & 1,8 & 5,7 \\
\hline Drechslera sp. & 5,4 & 0,3 & 1,8 & 0,0 & 3,9 & 19,0 & 11,0 & 0,7 & 0,0 & 0,0 & 6,7 & 12,8 \\
\hline Epicoccum sp. & 0,5 & 0,0 & 0,0 & 0,0 & 0,4 & 0,8 & 0,0 & 0,0 & 0,0 & 0,0 & 1,0 & 0,5 \\
\hline Fusarium sp. & 1,9 & 1,5 & 0,1 & 0,3 & 4,0 & 4,3 & 3,3 & 0,2 & 0,0 & 0,0 & 4,8 & 5,3 \\
\hline Penicillium sp. & 0,3 & 0,0 & 0,0 & 0,0 & 1,3 & 0,5 & 2,2 & 0,0 & 0,0 & 0,0 & 2,8 & 2,7 \\
\hline Phoma sp. & 0,5 & 0,8 & 0,3 & 0,0 & 5,3 & 7,4 & 5,2 & 0,8 & 0,0 & 0,0 & 6,0 & 10,2 \\
\hline Rhizopus sp. & 0,4 & 0,5 & 0,0 & 0,1 & 2,6 & 1,1 & 2,8 & 0,0 & 0,0 & 0,0 & 3,6 & 1,3 \\
\hline
\end{tabular}

TABELA 6 - Coeficientes de correlação simples entre as combinações das variáveis estudadas para B.brizantha, lote 1 (1) e lote 2 (2). Piracicaba, 1989.

\begin{tabular}{|c|c|c|c|c|c|c|}
\hline & \multicolumn{2}{|c|}{$\begin{array}{l}\text { \% Incidência fungos } \\
\text { teste sanidade }\end{array}$} & \multicolumn{2}{|c|}{$\begin{array}{l}\text { \% Incidência fungos - } \\
\text { teste germinação - } 10 \text { dias }\end{array}$} & \multicolumn{2}{|c|}{$\begin{array}{l}\text { \% Incidência fungos - teste } \\
\text { germinação - } 21 \text { dias }\end{array}$} \\
\hline & (1) & (2) & (1) & (2) & (1) & (2) \\
\hline \% Germinação & $-0,30^{\text {ss }}$ & $-0,14^{m}$ & $-0,52^{-}$ & $-0,22^{\mathrm{ss}}$ & $-0,63^{-}$ & $-0,40^{m}$ \\
\hline $\begin{array}{l}\text { \% Incidência fungos } \\
\text { teste sanidade }\end{array}$ & - & - & $0,83^{*}$ & $0,83^{* *}$ & $0,79^{-\infty}$ & $0,78^{*}$ \\
\hline $\begin{array}{l}\text { \% Incidência fungos } \\
\text { teste germinação - } \\
10 \text { dias }\end{array}$ & - & - & - & - & $0,95^{*}$ & $0,91^{--}$ \\
\hline
\end{tabular}

ns $=$ não significativo

** = significativo ao nível de $1 \%$ de probabilidade

URBEN (1987), trabalhando com sementes de braquiária, constatou que o método do papel de filtro proporcionou maior recuperação de fungos em relação a outros métodos utilizados.

É interessante ressaltar, ainda, que no lote 1 verificou-se incidência mais pronunciada de Drechslera spp. e de Fusarium sp.; estes fungos também se destacaram nas sementes de braquiária, em trabalho realizado por CHAGAS \& OLIVEIRA (1983). A elevada ocorrência de Drechslera spp., nos dois lotes, vem confirmar as afirmações feitas por NEERGAARD (1977) de que este patógeno apresenta notável afinidade por gramíneas.

A TABELA 6 contém os coeficientes de correlação entre os parâmetros estudados. Para o lote 1 , as correlações entre porcentagem de germinação e porcentagem de incidência de fungos aos 10 e 21 dias foi negativa e altamente significativa, indicando que reduções no poder germinativo estão diretamente associadas a 
elevações nos níveis de incidência de fungos. Este fato vem reforçar o registro de que o tratamento 6 deste lote foi inferior aos demais.

No lote 2, não houve correlação significativa entre germinação e incidência de fungos em todas as avaliações feitas. No entanto, foram observados coeficientes positivos e altamente significativos entre as avaliações envolvendo determinação da incidência de fungos. Tais fatos mostram que o uso do teste de germinação para avaliar a sanidade de sementes de braquiária, em casos eventuais, pode fornecer informações complementares, principalmente em situações de impossibilidade de execução do teste de sanidade.

\section{CONCLUSÕES}

- A escarificação com ácido sulfúrico não promoveu acréscimo significativo na germinação e contribuiu para a redução do nível de incidência de fungos no teste de germinação;

- Os fungicidas testados foram eficientes para reduzir a incidência de microrganismos nos testes de germinação e facilitaram a interpretação e a contagem das plântulas;

- Foram identificados os seguintes fungos: Alternaria tenuis, Aspergillus spp., Cladosporium sp., Curvularia sp., Drechslera spp., Epicoccum sp., Fusarium sp., Neurospora monilia, Penicillium sp., Phoma sp., Rhizopus sp., Trichoconiella padwikii e Trichothecium sp.; eventualmente, a avaliação sanitária do teste de germinação poderá substituir o teste de sanidade.

\section{REFERÊNCIAS BIBLIOGRÁFICAS}

ANDERSEN, A.M. A germination study of Merion Kentucky bluegrass. Proceedings of the Association of Official Seed Analysis, Washington, v.45, p.94 101, 1955.

ANDERSEN, A.M. The effect of certain fungi on the germination of Merion Kentucky bluegrass seed. Proceedings of the Association of Official Seed Analysis, Washington, v.47, p.145-153, 1957.

AUSTRÁLIA, Queensland Department of Primary Industries. Seed Testing Laboratory. Seed testing procedures. Bresbane : Queensland, Department of Primary Industries, 1970. 33p.
BARNETT, H.L. \& HUNTER, B.B. Ilustrated genera of imperfect fungi. 3. ed. Minneapolis : Burgess Pub., 1972. 241p.

CHAGAS, D.; OLIVEIRA, D.P. Fungos associados a sementes de gramíneas e leguminosas forrageiras. Fitopatologia Brasileira, Brasília, v.8, n.1, p.131-135, 1983.

DAVIDSON, D. E. Five pasture plants for Queensland. Queensland Agricultural Journal, Brisbane, v.92, p.460-466, 1966 .

GOEDERT, C. O. Efeitos de reagentes químicos na superação da dormência em sementes de gramíneas forrageiras. In: CONGRESSO BRASILEIRO DE SEMENTES, 4., 1985, Brasília, Resumos... Brasília, ABRATES, 1985 . p.66.

GROF, B. Viability of seed of Brachiaria decumbens. Queensland Journal of Agricultural and Animal Sciences, Brisbane, v.25, p.149-152, 1968.

INTERNATIONAL SEED TESTING ASSOCIATION. International rules for seed testing. Seed Science \& Technology, Zurich, v.13, n.2, p.299-355, 1985.

JARK FILHO, W. Estudo sobre a quebra de dormência em sementes de Brachiaria decumbens Stapf. Piracicaba, 1976, 63p. Dissertaçāo (Mestrado) - Escola Superior de Agricultura "Luiz de Queiroz", Universidade de São Paulo.

KONDE, B.K.; DHAGE, B.V.; MORE, B.B. Laboratory evaluation of pesticides for the control of seed-borne fungi of pearl millet. Pesticides, Bombay, v.18, n.11, p.36-39, 1984.

LUTRELL, E.S.; CROWDER, L.V.; WELLS, H.D. Seed treatment tests with pearl millet sudan grass, and browntop millet. Plant Disease Reporter, Washington, v.39, n.10, p.756-761, 1955.

McGEE, D.C. Penicillium contamination of seed grass seed germination tests. Journal of Seed Technology, Lansing, v.4, n.2, p.18-23, 1979.

MCLEAN, D.; GROF, B. Effect of seed treatments on Brachiaria mutica and Brachiaria ruziziensis. Queensland Journal of Agricultural and Animal Sciences, Brisbane, v.25, p.81-85, 1968.

MENDES, M.A.S.; MARQUES, A.S.A.; URBENS, A.F.; MARINHO, V.L.A.; PARENTE, P.M.G.; FONSECA, J.N.L. Patógenos associados a germoplasma vegetal interceptados pela quarentena de pós-entrada no Brasil. In: CONGRESSO BRASILEIRO DE SEMENTES, 6., 1989, Brasília, 
Resumos... Brasilia, ABRATES : 1989.p. 105.

NEERGAARD, P. Seed pathology. London : Macmillan, 1977. 2v.

ORTOLANI, D.B. Campinas, Coordenadoria de Assistência Técnica Integral, Divisāo de Sementes Mudas e Matrizes, 1989, p. ，(CATI. Carta Circular 092-89).

PANDY, K.N.; PARDE, B.C.; GUPTA, R.C. Efficacy of some fungicides on incidence of seed-borne fungi of Setaria italica grow in Almora Hills. Madras Agricultural Journal, Coimbatore, v.68, n.2, p.8689,1981

PANDY, M.L. \& GUPTA, R.C. Effect of fungicidal treatments on seed mycoflora and germination of Setaria tlalica in Kaumaun Hills. Madras Agricultural Joumal, Madras, v.71, n 9, p.599-602, 1984.

SHETTY, H.S., MATHUR, S B ; NEERGAARD, P.; SAFEEULLA, K. M. Diechslera setane in Indian pearl millet seeds, its seed borne nature, transmission and significance. Transaction British Mycological Society, London, v 78, n 1, p.170-173, 1982

TANAKA, M A S.; PAOLINELLI, G.P. Avaliação sanitária a fisiológica de sementes de algodão produzidas em Minas Girals Revista Brasileira de Sementes, Brasilia, v.6, n 1, p 71-81, 1984.
TOLEDO, F.F. Germinação e armazenamento de sementes de capim colonião tratadas com fungicidas comerciais. In: REUNIĀO ANUAL DA SOCIEDADE BRASILEIRA PARA O PROGRESSO DA CIÊNCIA, 29., 1977, São Paulo. Resumos... São Paulo : SBPC, 1977. p.22.

TOSEllo, J.; ATALlA, L.M.O. Germinação de sementes de Brachiaria. s.l. Campinas, CATI, 1978. (CATI - Informativo, 12). s.p.

URBEN, A.F. Testes de sanidade em sementes de forrageiras. In: SOAVE, J.; WETZEL, M.M.V.S. (Ed.) Patologia de sementes. Campinas, Fundação Cargill, 1987. p.406-429.

WHITEMAN, P.C.; MENDRA, K. Effects of storage and seed treatments on germination of Brachiana decumbens. Seed Science \& Technology, Zurich, v.10, n.2, p 233-242, 1982.

Trabalho entregue para publıcação em 11.05 .92

Trabalho aprovado para publicaçāo em 22.0792 